\title{
Features of Scattering of a Plane Electromagnetic Wave on a Conductive Ball
}

\author{
A.V. Sargsyan \\ Institute of Applied Problems of Physics NAS RA, 25 Hr. Nersessian St., Yerevan, Armenia \\ E-mail: anush.sarg@gmil.com
}

Received: November 6, 2021; Revised: November 24, 2021; Accepted: November 30, 2021

\begin{abstract}
The phenomenon of scattering of a plane electromagnetic wave on a nonmagnetoactive conductive ball is studied. The dispersion of electromagnetic waves inside the ball material is taken into account. A special case of that phenomenon (the radius of the ball is much smaller than the wavelength of the scattered wave) lies in the basis of the work of a spaser, which can have a wide range of important practical applications.
\end{abstract}

Keywords: electromagnetic waves, nonmagnetoactive ball, scattering, nanoparticle

https://doi.org/10.54503/18291171-2021.14.4-173

\section{Introduction}

The interaction between electromagnetic waves and small spherical metal particles is under consideration for years (see e.g. [1]). Our particular interest is the case, when the particle is smaller, than the wavelength of scattered wave.

Consider scattering of a plane electromagnetic wave on a conductive ball. Our goal is to study the most apparent features of the scattered field, caused by the falling wave frequency and by the choice of the conductive ball parameter values. We study the electromagnetic waves at large distances. The analysis is based on the corresponding exact analytical solutions of Maxwell equations, using vector spherical functions. The medium is non-homogeneous, spherically-symmetric and the solutions are obtained by the method of Green function [2, 3].

The importance of that phenomenon is their usage in many electronic devices, such as lasers, nanolasers. The first sample of nanolaser had been presented in 2008 in USA, and the researchers got the Nobel Prize [4]. That could become the main component of future optical computers. Researchers are especially concentrating on the fact, that due to the very small size of metal nanostructures and the optical speed of the processes occurring in them, nanoplasmonics will create a new element base for optical computers and data processing devices. There is a case, when one can obtain high intensity radiation. For instance, the operation of spaser [5] is based on special case of this phenomenon under consideration, when the ball radius is much smaller than the wavelength of scattered wave. It can be used to amplify surface waves.

The paper provides the basis of the theory of the characteristic features of this phenomenon and its obvious explanation.

\section{Electromagnetic waves in a spherically symmetric medium}

\subsection{Lorenz gauge}

Let us consider the scattering of electromagnetic waves in a spherically symmetric and inhomogeneous medium. It is convenient to go over to Fourier expansions of the physical quantities: 


$$
F=\frac{1}{\sqrt{2 \pi}} \int F_{\omega} e^{-i \omega t} d \omega, F=\vec{E}, \vec{H}, \vec{A}, \varphi .
$$

Let us introduce scalar $\varphi$ and vector $\vec{A}$ potentials [6-8]

$$
\vec{E}_{\omega}=-\vec{\nabla} \varphi_{\omega}+\frac{i \omega \varepsilon}{c} \vec{A}_{\omega}, \quad \vec{B}_{\omega}=\vec{\nabla} \times \vec{A}_{\omega}
$$

In the Lorentz gauge, the relationship between scalar $\varphi_{\omega}$ and vector $\vec{A}_{\omega}$ potentials is described by equation

$$
\operatorname{div} \vec{A}_{\omega}=-i \varepsilon \frac{\omega}{c} \varphi_{\omega}
$$

We choose a spherical coordinate system $r, \theta, \varphi$ with the origin at the center of symmetry of the medium. As a result, the dielectric $(\varepsilon)$ and magnetic $(\mu)$ permittivities of the medium will depend only on the quantities $r$ and $\omega$ and will not depend on $\theta, \varphi$ :

$$
\varepsilon=\varepsilon(r, \omega), \quad \mu=\mu(r, \omega)
$$

In a nonmagnetoactive medium with $\mu=1$ the system of Maxwell equations is transformed into a single vector equation that describes the propagation of electromagnetic waves in a spherically symmetric and inhomogeneous medium [8]

$$
\left(\Delta+\frac{\omega^{2}}{c^{2}} \varepsilon\right) \vec{A}(\vec{r})-\frac{1}{\varepsilon}(\vec{\nabla} \varepsilon) \operatorname{div} \vec{A}(\vec{r})=0 .
$$

Here $\varepsilon$ can be an arbitrary function depending on $r$ and $\omega$. Thus, solving the differential equation (5), one can determine the propagation of electromagnetic waves for any given function $\varepsilon(r, \omega)$.

\subsection{Selection of basis vector functions}

In our case (a spherically symmetric medium) it is convenient to choose a basis of so-called vector spherical functions $\vec{X}_{l m}{ }^{(\mu)}$ (spherical vectors) [9, 10].

An arbitrary vector field $\vec{S}$ can be expanded in a series over these vectors $\vec{X}_{l m}{ }^{(\mu)}$ :

$$
\vec{S}(\vec{r})=\sum_{\mu=1}^{3} \sum_{l=0}^{\infty} \sum_{m=-l}^{l} S_{\mu}^{l m}(r) \vec{X}_{l m}{ }^{(\mu)}(\Omega)
$$

where $\vec{X}_{l m}{ }^{(\mu)}$ is the vector spherical function of longitudinal ( $\left.\mu=1\right)$, electric $(\mu=2)$, and magnetic $(\mu=3)$ types [9].

According to (6)

$$
\vec{A}(\vec{r})=\sum_{\mu=1}^{3} \sum_{l=0}^{\infty} \sum_{m=-l}^{l} A_{\mu}{ }^{l m}(r) \vec{X}_{l m}{ }^{(\mu)}(\Omega)
$$

the determination of a vector field $\vec{A}(\vec{r})$ reduces to the determination of functions $A_{\mu}^{l m}(r)$ that do 
not depend on the solid angle $\Omega=(\theta, \varphi)$. Substituting (7) in (5) and multiplying the both sides of this equation by $\vec{X}_{l m}{ }^{(\mu)^{*}}$, after integration by $\Omega=(\theta, \varphi)$ with respect to the orthonormality of the vector functions $\vec{X}_{l m}{ }^{(\mu)}$ [8], one finds that [11,12]

$$
\sum_{v=1}^{3}\left[F_{\mu \nu}(r ; l)+\frac{\omega^{2}}{c^{2}} \varepsilon \delta_{\mu \nu}-\frac{1}{\varepsilon} \frac{\partial \varepsilon}{\partial r} D_{\mu \nu}(r ; l)\right] A_{\nu}^{l m}(r)=0 .
$$

Here $\hat{F}$ and $\hat{D}$ are $3 \times 3$ matrices and, at the same time, operators of second-order differentiation according to $r$. Their explicit forms are given in [11, 12].

Thus, the problem reduces to determining the function $A_{\mu}{ }^{l m}(r)$ from equation (8). One again notes that we replaced the solution of the basic vector equation (5) with the solution of the new basic equation (8). Its advantage lies in the fact that unknown functions $A_{\mu}^{l m}(r)$ depend only on one variable $r$.

\subsection{The solution of the fundamental equation (8)}

We will use the method [2,3,11-13] specially adapted to the solution of problems of electrodynamics in spherically symmetric layered media. In the framework of this method, the solution of equation (8) can be represented in the following form [13]

$$
\hat{A}(r)=\hat{A}_{(0)}(r)+\int_{0}^{\infty} \hat{G}^{(0)}(r, x ; l) \cdot \hat{V}(x, l) \cdot \hat{A}(x) d x,
$$

where

$$
\hat{V}(r, l)=\frac{1}{\varepsilon(r)} \frac{\partial \varepsilon}{\partial r} \hat{D}(r ; l)
$$

and the auxiliary functions $A_{\mu(v)}{ }^{l m}(r)$ are determined from the following "truncated" equations

$$
\sum_{v=1}^{3}\left[F_{\mu \nu}(r ; l)+\frac{\omega^{2}}{c^{2}} \varepsilon \cdot \delta_{\mu \nu}\right] A_{v(0)}^{l m}(r)=0 .
$$

They can be written in the following matrix form

$$
\left(\hat{F}+\frac{\omega^{2}}{c^{2}} \varepsilon \cdot \hat{I}\right) \hat{A}_{(0)}=0 .
$$

The Green function in (9) must satisfy the following truncated equation

$$
\left(\hat{F}+\frac{\omega^{2}}{c^{2}} \varepsilon \cdot \hat{I}\right) \hat{G}^{(0)}=\hat{I} \delta\left(r-r^{\prime}\right)
$$

Thus, the solution of the basic equation (8) reduces to solving the integral equation (9). The effectiveness of this method is due to the fact that in our case, if we substitute (15) in (10), then we get that 


$$
\hat{V} \sim \delta\left(r-r_{b}\right)
$$

As a result, the integral equation (9) reduces to a much simpler algebraic equation [11,12].

\section{Scattering of a plane electromagnetic wave on a conductive ball}

Now consider the propagation of electromagnetic waves, when a spherically symmetric medium is a nonmagnetoactive conductive ball surrounded by a vacuum.

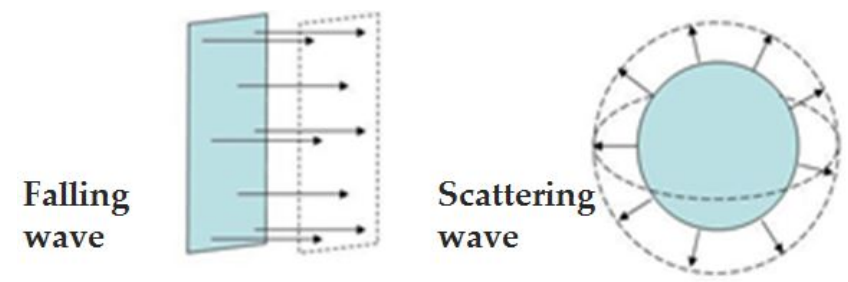

Fig. 1. Scattering of a plane electromagnetic wave on a conductive ball.

In this case, the dielectric permeability of the medium is a step function:

$$
\varepsilon(r)=\varepsilon_{b}+\left(\varepsilon_{0}-\varepsilon_{b}\right) \Theta\left(r-r_{b}\right)
$$

where $\Theta(x)$ is the Heaviside step function.

\subsection{Resonant scattering on a small size conductive ball}

If the dielectric permeability $\varepsilon$ of the medium accepts finite values and depends on the frequency $\varepsilon(\omega)$, then it turns out that in scattered electromagnetic wave spectra for certain frequencies there is a strong (resonant) scattering and it is particularly significant that it happens at frequencies $\omega_{0}$ when dielectric permeability of a small size ball is $\varepsilon=-2$.

In order to clarify the essence of this phenomenon, it is expedient to study the radiation that is generated when the charged particle uniformly passes through a center of a conductive ball.

\subsection{The strong (resonant) effect of the conductive ball on the charged particle radiation [14]}

Consider a charged particle that uniformly passes through the center of a conductive ball flying rectilinearly and uniformly (see Fig. 2).

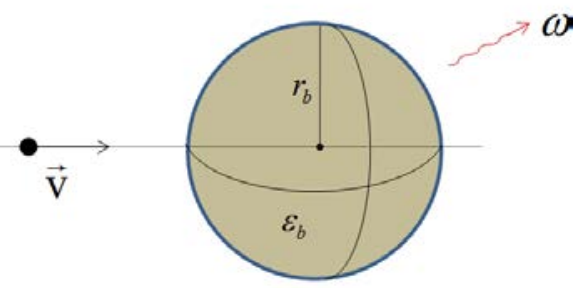

Fig. 2. Charged particle uniformly passes through the center of a conductive ball.

In this case, the dielectric permeability of the medium is a step function (15).

Let us investigate the spectral energy distribution 


$$
W=\int_{0}^{\infty} I(\omega) d \omega
$$

radiated by a charged particle, during its entire time of motion. An analogous problem for a particle passing through the center of a dielectric ball was solved in the work [15]. At the same time, the results of analytical calculations [15] are applicable without restrictions on values of $\varepsilon_{b}$ and the dependence of this quantity on the frequency $\omega$. Therefore, the expression for the spectral density of the radiated energy $I(\omega)$ derived in this paper is applicable also in the case of a conductive ball. The analytic expressions defining the explicit form of the function $I(\omega)$ (see [15]) are rather cumbersome, and therefore we do not give them here for brevity.

\subsection{Numerical results}

Numerical calculations were carried out [14] for a microscopic ball with a radius of $100 \mathrm{~nm}$ made of gold. The energy of a charged particle passing through the ball was assumed to be $2 \mathrm{MeV}$.

Comparing the given data (see Fig. 3), we arrive at the following conclusions:

In the emission spectrum of an electron there is a peak in the vicinity of a certain so-called "resonant frequency".

The value of the spectral density of the radiated energy at this frequency is (significant) many times more than the values of this quantity at nearby neighboring frequencies.

\subsection{The visual explanation}

The dotted curve in Fig. 3 describes the radiation of the same electron passing through a flat parallel plate made of gold and having a thickness equal to the diameter of the ball of $200 \mathrm{~nm}$. In both cases, the radiation is connected with the generation of electromagnetic oscillations in the ball material by a charged particle. The volume of the ball is much smaller than the volume of the plate and it is therefore natural to expect that the radiation in the case of the ball should be much weaker than in the case of the plate. In Fig 3 we see that this circumstance is confirmed, besides for the range around $4 \cdot 10^{15} \mathrm{~Hz}$ frequency.

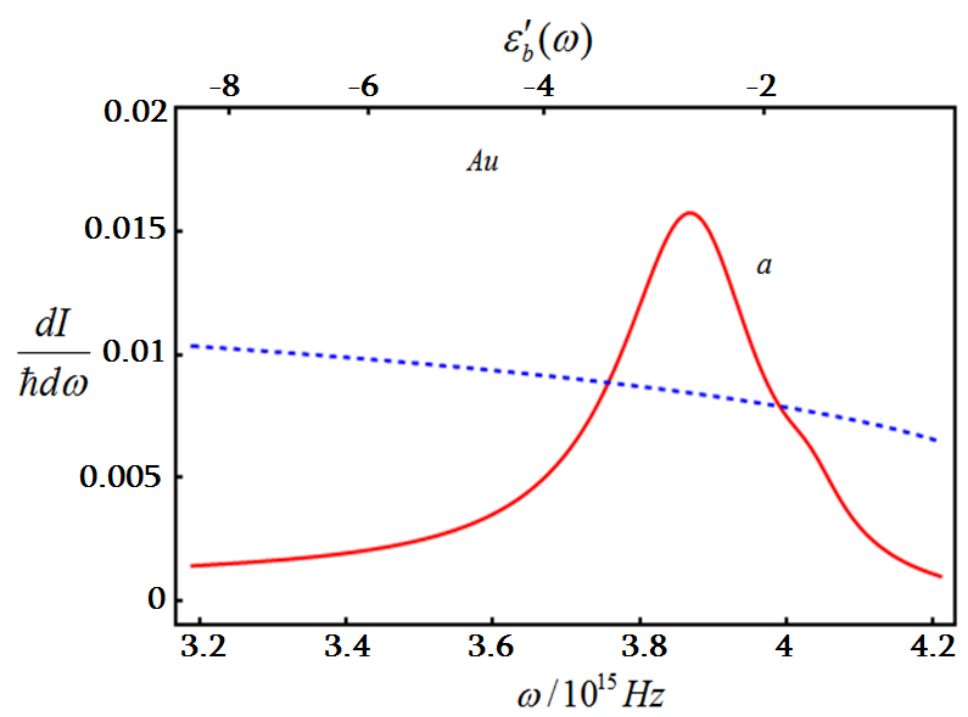

Fig. 3. The spectral distribution of radiation, generated by an electron passing through a golden ball, with radius of $100 \mathrm{~nm}$ (solid curve), and passing through a plane parallel golden plate, 
with the thickness equal to the diameter of the ball (dashed curve).

The course of the curves in Fig. 3 confirms this circumstance with the exception of a narrow frequency region near the resonant frequency of the order of $4 \cdot 10^{15} \mathrm{~Hz}$. The question arises: why near the resonant frequency of the order of $4 \cdot 10^{15} \mathrm{~Hz}$ the spectral density of the radiated energy in the case of the ball is greater than in the case of the plate? The key to the answer to this question is given in the upper part of Fig 3. The abscissa indicates the values of $\varepsilon_{A u}^{\prime}$. As can be seen from the given data, the real part of the dielectric permeability of the conductive ball at the resonant frequency assumes negative values $\varepsilon_{A u}^{\prime}=-2.4<0$.

In such a case, the dispersion equation forbids the propagation of electromagnetic waves in the ball material at least along one of the three independent directions in the ball, but it is possible the propagation of electromagnetic oscillations of a substance localized at the interface with a vacuum (surface waves). It is clear that surface waves are generated both in the case of the ball and in the case of the plate, but with one important difference. In the case of the plate, the interface with vacuum is infinite and, in the case of the ball, it is finite and closed, and therefore surface waves are superimposed on each other. At most frequencies there is a destructive superposition of electromagnetic oscillations on each other, as a result of which their amplitude decreases. However, at some frequencies (eigen frequencies of the ball), there is a constructive superposition of the oscillations on each other, as a result of which their amplitude significantly increases.

In summary, we reach the following conclusion: a charged particle, crossing the ball, generates surface electromagnetic oscillations at the eigen frequencies of the ball, which at large distance from this ball manifest themselves as resonant radiation.

\subsection{An appearing explanation of resonant scattering}

It is noteworthy that resonant scattering of a plane electromagnetic wave on a small size ball appears at the frequencies for which the value of the dielectric permeability is $\varepsilon=-2$, which is close to the value that provided in the case of resonant radiation. In this case falling electromagnetic wave generates surface waves on the ball, which superpose with each other on the resonance frequency coherently. These surface waves are accompanied by high intensive electromagnetic waves that propagate at a large distance from the ball. These waves are interpreted as resonant radiation in the case of falling electron and, in the case of a wave, as resonant scattering.

\section{Applications}

This resonant scattering may have important practical applications, such as in optronics [5]. It is noteworthy, that resonance scattering is used not only in spacer (in nanolaser), but it can also be used to determine the level of atmosphere pollution, particularly, by looking at its own resonance frequency, you can know what metal microparticles are for example in the current atmosphere.

\section{Conclusions}

The phenomenon of scattering of an electromagnetic wave on a nonmagnetoactive conductive ball is studied taking into account the dispersion of electromagnetic waves inside the ball material. The analysis is based on the corresponding exact analytical solutions of Maxwell equations. These solutions are obtained by the method of Green function presented in [2,3]. The visual explanation is given about why the resonant scattering may appear when plane waves of certain frequencies scatter on the ball. The operation of spacer [5] is based on the special case of the phenomenon under consideration, when the ball radius is much smaller than the wavelength of scattered wave. This phenomenon may have a wide spectrum of important practical applications. 


\section{Acknowledgement}

I would like to express my sincere gratitude to my advisor Prof. Levon Grigoryan and Dr. Hrant Khachatryan for the continuous support of my research, for their patience, motivation, enthusiasm and immense knowledge.

\section{References}

[1] I.A. Kuznetsova, M.E. Lebedev, A.A. Yushkanov, Tech. Phys. Lett. 40 (2014) 353.

[2] Y. Avishai, Y.B. Band, Phys. Rev. A 40 (1989) 5500.

[3] A.A. Bohm, Quantum Mechanics: Foundations and Applications (The World, Moscow, 1990).

[4] T. Hovhannisyan, Expert, №32(669), (2009) https://expert.ru/expert/2009/32/.

[5] D.J. Bergman, M.I. Stockman, Phys. Rev. Lett. 90 (2003) 027402.

[6] L.D. Landau, E.M. Lifshitz, The Classical Theory of Fields (Pergamon Press, New York, 1971).

[7] L.D. Landau, E.M. Lifshitz, Electrodynamics of Continuous Media (Nauka, Moscow, 1980).

[8] G.M. Garibian, Yan Shi, X-Ray Transition Radiation (AN Arm. SSR Press, Yerevan, 1983).

[9] V.B. Berestetskii, E.M. Lifshitz, L.P. Pitaevskii, Quantum Electrodynamics (Nauka, Moscow, 1980).

[10] J.D. Jackson, Classical Electrodynamics (John Willey \& Sons, New York-London, 1962).

[11] A.R. Mkrtchyan, L.Sh. Grigoryan, A.A. Saharian, S.R. Arzumanyan, Preprint IAPP NAS RA 2-91 (Yerevan, 1991).

[12] S.R. Arzumanyan, L.Sh. Grigoryan, A.A. Saharian, J. Contemp. Phys. (Arm. Acad. Sci.) 30 (1995) 99.

[13] L.Sh. Grigoryan, H.F. Khachatryan, A.V. Sargsyan, P.H. Mosoyan, Book of Abstract of the Intern. Conf. on "Electron, Positron, Neutron and X-Ray Scattering under External Influences” (2019) 25.

[14] L.Sh. Grigoryan, A.H. Mkrtchyan, H.F. Khachatryan, Proc. of the Intern. Conf. on "Electron, Positron, Neutron and X-Ray Scattering under External Influences" (2016) 47.

[15] S.R. Arzumanyan, J. Phys.: Conf. Series 357 (2012) 012008. 